\title{
Variation in post-traumatic response: the role of trauma type in predicting ICD-11 PTSD and CPTSD symptoms
}

\author{
Philip Hyland ${ }^{1,2}$ (D) Jamie Murphy ${ }^{3} \cdot$ Mark Shevlin $^{3} \cdot$ Frédérique Vallières $^{2} \cdot$ \\ Eoin McElroy $^{3} \cdot$ Ask Elklit $^{4} \cdot$ Mogens Christoffersen $^{5} \cdot$ Marylène Cloitre $^{6,7}$
}

Received: 26 September 2016 / Accepted: 22 January 2017 / Published online: 14 February 2017

(C) Springer-Verlag Berlin Heidelberg 2017

\begin{abstract}
Purpose The World Health Organization's 11th revision to the International Classification of Diseases manual (ICD-11) will differentiate between two stress-related disorders: PTSD and Complex PTSD (CPTSD). ICD-11 proposals suggest that trauma exposure which is prolonged and/or repeated, or consists of multiple forms, that also occurs under circumstances where escape from the trauma is difficult or impossible (e.g., childhood abuse) will confer greater risk for CPTSD as compared to PTSD. The primary objective of the current study was to provide an empirical assessment of this proposal.

Methods A stratified, random probability sample of a Danish birth cohort (aged 24) was interviewed by the Danish National Centre for Social Research $(N=2980)$ in
\end{abstract}

Philip Hyland

Philip.hyland@ncirl.ie

1 School of Business, National College of Ireland, IFSC, Mayor Street, Dublin 1, Ireland

2 Centre for Global Health, School of Psychology, Trinity College Dublin, College Green, Dublin 2, Ireland

3 Psychology Research Institute, School of Psychology, Ulster University, Northland Road, Co. Londonderry, Londonderry BT48 7JL, Ireland

4 National Centre for Psychotraumatology, Institute for Psychology, University of Southern Denmark, Campusvej 55, 5230 Odense M, Denmark

5 The Danish National Centre for Social Research, Copenhagen, Denmark

6 National Centre for PTSD Division of Dissemination and Training, Department of Psychiatry and Behavioural Sciences, Stanford University, Stanford, CA, USA

7 Department of Psychiatry and Behavioral Science, Stanford University, Palo Alto, CA, USA
2008-2009. Data from this interview were used to generate an ICD-11 symptom-based classification of PTSD and CPTSD.

Results The majority of the sample (87.1\%) experienced at least one of eight traumatic events spanning childhood and early adulthood. There was some indication that being female increased the risk for both PTSD and CPTSD classification. Multinomial logistic regression results found that childhood sexual abuse $(\mathrm{OR}=4.98)$ and unemployment status $(\mathrm{OR}=4.20)$ significantly increased risk of CPTSD classification as compared to PTSD. A dose-response relationship was observed between exposure to multiple forms of childhood interpersonal trauma and risk of CPTSD classification, as compared to PTSD.

Conclusions Results provide empirical support for the ICD-11 proposals that childhood interpersonal traumatic exposure increases risk of CPTSD symptom development.

Keywords Posttraumatic stress disorder (PTSD) ·

Complex-PTSD (CPTSD) · ICD-11 · Childhood trauma .

Childhood sexual abuse

\section{Introduction}

The 11th revision to the International Classification of Diseases (ICD-11) manual, due for publication in 2018 by the World Health Organization, will include a revised conceptualisation of trauma-related psychopathology. The ICD-11 will include two related, but distinct trauma-based disorders: posttraumatic stress disorder (PTSD) and Complex PTSD (CPTSD: Maercker et al. [1]). The guiding principle underlying revisions for ICD-11 is to simplify disorder classification, where possible by focusing on the core constituent symptoms, so as to improve clinical utility worldwide 
[2]. Following the work of Brewin, Lanius, Novac, Schnyder, and Galea [3], the ICD-11 symptom profile of PTSD will be narrower than any previous description comprising of six, or possibly seven, symptoms (n.b., a seventh symptom may be included for those trauma survivors that do not possess a clear memory of the traumatic event to which they were exposed). These symptoms relate to three essential symptom clusters: (1) re-experiencing of the traumatic event in the here and now (Re: two symptoms, or three symptoms if one does not possess a clear memory of the trauma), (2) active avoidance of reminders of the trauma (Av: two symptoms), and (3) a heightened sense of current threat (Th: two symptoms).

Following the work of Herman [4], CPTSD is proposed as a broader clinical disorder that includes the core PTSD symptoms but is conceptually distinguishable from PTSD on the basis of symptoms that reflect 'disturbances in selforganization' (DSO). These DSO symptoms reflect three essential symptom clusters: (1) affective dysregulation (AD), (2) negative self-concepts (NSC), and (3) disturbances in relationships (DR). These additional DSO clusters reflect the pervasive psychological disturbances that can occur following traumatic exposure, across a variety of contexts, and even in the absence of traumatic reminders. The symptom groupings were selected on the basis of the symptoms that were most frequently reported by participants during the DSM-IV field trials assessing CPTSD [5], along with those symptoms identified as the most frequent and most impairing by expert clinicians in a consensus survey on CPTSD [6]. Presently, the exact number of DSO symptoms to be included in the ICD-11 symptom profile for CPTSD has not been finalized; however, in line with the guiding principles of ICD-11, the likelihood is that each DSO cluster (AD, NSC, and DR) will include 2-3 symptoms.

ICD-11 requires exposure to a traumatic event as a gateway for a diagnosis of either PTSD or CPTSD. With respect to a differential diagnosis between PTSD and CPTSD, the nature of the traumatic stressor is considered a risk factor rather than a requirement for a differential diagnosis; this is to allow recognition of the role played by genetic and environmental risk and resiliency factors (e.g., social support) in the development of distinct traumatic responses. It is proposed that exposure to interpersonal traumas that are prolonged and repeated in nature, or comprise multiple forms, from which escape is difficult or impossible, will increase the risk of DSO symptomatology in addition to the core PTSD symptoms $[4,7,8]$. Although CPTSD responses can occur following these types of events in adulthood (e.g., torture experiences, prisoner of war experiences, repeated combat exposures), exposure to repeated, prolonged, and multiple forms of interpersonal trauma during early developmental periods are proposed to be among those that are most strongly associated with a CPTSD response [9]. This proposal is derived from an extensive literature indicating that exposure to severe interpersonal maltreatment during childhood can impair normal development of emotional regulatory capacities, increase dysfunctional beliefs about oneself, and lead to problematic interpersonal functioning [10-12], and can have a long-term impact in adulthood [13]. Childhood abuse is a prototypical example of a risk factor for CPTSD as it is frequently prolonged, repeated, and often comprised of multiple forms of interpersonal trauma (e.g., sexual, physical, and verbal abuse) $[14,15]$. In addition, abuse during childhood is difficult to escape from due to maturational, psychological, social, and environmental constraints.

The existing literature regarding the relationship between exposure to sustained childhood interpersonal trauma and risk of ICD-11 CPTSD is limited and somewhat contradictory. For example, a study of treatment-seeking men and women found that those who had experienced repeated childhood abuse or multiple types of interpersonal violence were more likely to have a CPTSD profile [7]. This finding has not been replicated when childhood abuse has been defined as a single categorical "yes/no" variable [16] or when "sexual trauma" exposure does not include age [17]. These conflicting results may reflect a lack of sensitivity of the measures regarding potentially important aspects of the experience such as whether the experience was sustained or repeated, or whether it occurred in childhood or not. A similarly inconsistent picture exists with regards to another well-established risk factor in the psychotraumatology literature: sex. Perkonigg et al. [18] found that being female increased risk for both ICD-11 PTSD and CPTSD, while Hyland et al. [8] found that females were at an elevated risk for both disorders but that the risk was significantly greater for PTSD than for CPTSD. Contrastingly, Cloitre et al. [7] and Wolf et al. [17] found no sex-specific risk for either disorder.

While considerable evidence is accumulating to support the proposed competing symptom structures of both PTSD [19-21] and CPTSD [7, 8, 21, 22], as well as the qualitative distinctions between both disorders $[7,16,18$, $23,24]$, there remains insufficient evidence regarding the factors that may serve to distinguish CPTSD responses to trauma from PTSD responses to trauma, as per the ICD11 proposals. In fact, the empirical literature to date seems to consider much about how ICD-11 CPTSD is conceptually and symptomatically distinct from ICD-11 PTSD, but little about why. Given the growing empirical support for the construct validity of both disorders, it is important to begin to develop a more thorough understanding of the factors that can differentially predict a CPTSD response from a PTSD response. This reflects the primary aim of the current study and two hypotheses were thus formulated. 
First, given the composition of the DSO symptoms (AD, NSC, and DR) that uniquely characterise CPTSD, along with the well-established association between such symptomatology and childhood interpersonal trauma experiences, we predicted that childhood exposure (0-18 years) to sexual abuse, physical assault, and bullying, individually, would be associated with an increased likelihood of a complex traumatic response. Second, in-line with ICD-11 proposals, we predicted that exposure to multiple forms of childhood interpersonal trauma would be associated with an increased risk of CPTSD, as compared to PTSD, in a dose-response fashion. Finally, in addition to these hypotheses, we also examined the role of sex; however, given the inconsistent empirical findings to date no formal hypothesis was developed regarding the relationship between sex and traumatic response.

\section{Methods}

\section{Participants and procedures}

Data were collected as part of a national study conducted by The Danish National Centre for Social Research in 2008-2009 based on a stratified random probability sample of the entire birth cohort of Danes born in 1984. A total of 4718 Danes aged 24 years were contacted and 2980 agreed to be interviewed (response rate $=63 \%$ ). Participation in the interview was voluntary and the study was approved by the Danish Data Protection Agency. The data used in the current study were collected in accordance with the Helsinki Declaration of 1989. To increase the number of participants who had experienced childhood abuse and neglect, children who had been assigned a child protection service status by Danish authorities were oversampled using a 1:2 ratio $(n=850)$. To adjust for the over-sampling of child protection cases, the data have been weighted so that findings are representative of the total Danish population born in 1984. A structured interview was conducted by telephone, or residential visit if telephone contact could not be made (mean duration of interview $=43 \mathrm{~min}$ ). All participants received written contact prior to the interview informing them of the process of the interview, the nature of the questions, and confidentiality. Interviewers were trained and instructed by The Danish National Centre for Social Research and participated in test trials to become familiar with the questionnaire and the coding procedures. Due to the sensitive nature of many of the questions (e.g., childhood sexual abuse experiences), participants had the opportunity to speak to a psychologist, via telephone, after the interview.

The majority of the sample $(87.1 \%, n=2591)$ were exposed to at least one of the eight traumatic life events measured and thus satisfied the ICD-11's requirement for traumatic exposure as a pre-requisite for either a PTSD or a CPTSD diagnosis. All analyses in the current study were based on these trauma-exposed individuals. Among this trauma-exposed sub-sample, there were relatively equal numbers of males $(51.9 \%, n=1346)$ and females $(48.1 \%$, $n=1245$ ). Approximately, half of respondents were single $(53.4 \%, n=1382)$, while the remainder were married $(6.2 \%, n=160)$ or living with a partner $(40.5 \%, n=1049)$, and the majority of individuals did not have children (90.9\%, $n=2352)$. Just over one-quarter of the sample owned their own home $(28.6 \%, n=742)$ and the remainder lived in some form of rented accommodation. The majority of individuals were in some form of employment (93.8\%, $n=2429)$ and a minority were unemployed $(6.2 \%, n=160)$.

\section{Measures}

\section{Traumatic exposure}

The specific types of trauma exposure reflected the key predictor variables in this study. Individuals were asked to indicate if they had directly experienced eight traumatic events during different developmental periods (0-18 years of age, or after 18 years of age), death of a close family member, road traffic accident, near-drowning, being the victim of a robbery, physical assault during adulthood ( $>18$ years of age), physical assault during childhood ( $<18$ years of age), bullying during childhood ( $<18$ years of age), and sexual abuse during childhood $(<18$ years of age). Responses to all trauma types were coded as "No" $(0)$ or "Yes" (1).

\section{ICD-11 PTSD and CPTSD}

The outcome variables in the current study were algorithmdriven classifications for PTSD and CPTSD. As the survey was conducted prior to the introduction of the ICD11 proposals, a specific measure for these constructs was not used. A symptom profile as reflective as possible of the PTSD and CPTSD proposals (e.g., First et al. [2]) was developed based on various questions contained within the full interview. The interview contained a screening measure for PTSD which included one item measuring re-experiencing of the trauma ("experience of nightmares/intrusive thoughts about your worst trauma"), one item measuring avoidance ("experience of avoidance of reminders of the situation/recollections of your worst trauma"), and one item measuring a sense of threat ("experience of constant hypervigilance related to your worst trauma"). Responses to these questions were limited to "No" (0) or "Yes" (1). No question was included to measure functional impairment associated with these symptoms. An algorithm-driven 
classification of ICD-11 PTSD was assigned if an individual endorsed all three symptoms.

The DSO symptom clusters that comprise CPTSD were each assessed using two questions from the interview to reflect the proposed ICD-11 symptoms as closely as possible. To measure affective dysregulation, one item was selected from the PTSD screening measure ("experience of emotional detachment from others and surroundings") and one item was selected from a separate personality questionnaire ("are you easily angered and often hot-tempered"). To measure negative self-concept, two items were selected from the Rosenberg Self-Esteem Scale [25]: "I certainly feel useless at times" and "all in all, I am inclined to feel that I am a failure." To measure disturbances in relationships, one item was selected from a measure of self-concept ("do you often feel rejected by others") and the second item was selected from a measure of personality ("do you have trouble making friends"). All items were coded on a "No" (0) or "Yes" (1) basis. An algorithm-driven classification of ICD-11 CPTSD was made if an individual satisfied the PTSD symptom requirements and endorsed 1 of 2 symptoms from each of the three DSO clusters.

Three classes were consequently developed for analytical purposes: (1) individuals who were classified as meeting ICD-11 PTSD symptomatology only, (2) individuals who were classified as meeting ICD-11 CPTSD symptomatology, and (3) individuals who did not meet the symptom requirements for either PTSD or CPTSD. These three classes are henceforth referred to as the 'PTSD class', 'CPTSD class', and 'non-symptomatic class'.

\section{Statistical analysis}

The analysis for this study included four parts. First, the proportion of the sample belonging to the PTSD and CPTSD classes was estimated, along with assessments of sex differences in class membership. Sex differences with regards to exposure to the various traumatic events were also calculated. Second, Chi-square tests of independence were conducted to calculate unadjusted odds ratios (ORs) between each traumatic event and PTSD and CPTSD class membership, as compared to the non-symptomatic class. Unadjusted ORs between exposure to each traumatic event and CPTSD class membership, as compared to PTSD class membership, were additionally estimated. Third, multinomial logistic regression analysis was conducted to calculate adjusted ORs regarding the likelihood of PTSD and CPTSD class membership, as compared to the non-symptomatic class (the reference category), due to exposure to each of the eight traumatic stressors. A second multinomial logistic regression was conducted in which the PTSD class was set as the reference group to determine whether exposure to specific types of traumatic events was associated with CPTSD classification as compared to PTSD classification. In both sets of analyses, a number of covariates were included. Sex was included as a covariate for two purposes: (1) to assess the association between sex and PTSD and CPTSD classification, respectively, and (2) to adjust for sex differences in traumatic exposure so as to produce interpretable estimates of the associations between traumatic exposure and risk of PTSD and CPTSD classification, respectively. Living status $(0=$ owing one's own home, $1=$ living in rented accommodation) and employment status $(0=$ employed, $1=$ unemployed $)$ were also included as covariates due to the use of non-clinical assessments of DSO symptomatology, and due to the fact that the PTSD and DSO symptoms were not anchored to a specific traumatic event. Inclusion of these variables provides some control for the fact that the PTSD, and in particular the DSO symptoms, was measured in the absence of a traumatic anchor and thus may be experienced due to sociodemographic reasons (e.g., living in impoverished conditions) rather than due to traumatic exposure.

Fourth and finally, a multinomial logistic regression was conducted in which the PTSD class was again set as the reference group to determine whether cumulative childhood interpersonal traumatic exposure (sum of childhood physical assault, childhood bullying, and childhood sexual abuse) was associated with an increased risk of CPTSD classification in a dose-response fashion. This model included sex, living status, employment status, and each form of adult traumatic exposure as covariates.

\section{Results}

\section{Descriptive statistics}

In total, $3.0 \%$ of the sample were assigned to the PTSD class, and $1.0 \%$ were assigned to the CPTSD class. ${ }^{1}$ Females were significantly more likely that males to belong to the PTSD and CPTSD classes. The most commonly endorsed symptom cluster was Affective Dysregulation with half of the sample satisfying this criterion. Significant sex differences were evident on each symptom cluster with the exception of the Disturbed Relationship cluster (see Table 1 for full details).

Table 2 includes the frequency of exposure to each form of traumatic stressor. The most commonly reported forms of traumatic exposure were experiencing the death of a close family member, childhood physical assault,

\footnotetext{
${ }^{1}$ Note that no person is in both classes. In line with the taxonomic structure of the ICD, CPTSD is not a subtype of PTSD and therefore a person can only be diagnosed with one of the two disorders.
} 
Table 1 Proportion of total trauma-exposed sample, males, and females meeting criteria for each symptom cluster, and PTSD and CPTSD

\begin{tabular}{|c|c|c|c|c|}
\hline & $\begin{array}{l}\text { Total sample } \\
(N=2591) \\
\%(n)\end{array}$ & $\begin{array}{l}\text { Males }(N=1346) \\
\%(n)\end{array}$ & $\begin{array}{l}\text { Females }(N=1245) \\
\%(n)\end{array}$ & $Z(p)$ \\
\hline \multicolumn{5}{|l|}{ Symptom clusters } \\
\hline Re-experiencing & $22.0(569)$ & $16.6(250)$ & $24.8(354)$ & $5.95(<0.001)$ \\
\hline Avoidance & $14.2(368)$ & $10.5(163)$ & $15.9(226)$ & $4.35(<0.001)$ \\
\hline Sense of threat & $8.2(213)$ & $6.5(102)$ & $8.6(123)$ & $2.13(0.016)$ \\
\hline Affective dysregulation & $50.3(1301)$ & $44.6(692)$ & $52.7(749)$ & $4.40(<0.001)$ \\
\hline Negative self concept & $18.8(488)$ & $14.7(228)$ & $21.6(309)$ & $4.98(<0.001)$ \\
\hline Disturbed relationships & $19.6(508)$ & $18.3(284)$ & $18.4(262)$ & $0.09(0.466)$ \\
\hline \multicolumn{5}{|l|}{ Class membership rates } \\
\hline Non-symptomatic class & $96.0(2487)$ & $97.4(1515)$ & $95.2(1356)$ & - \\
\hline ICD-11 PTSD class & $3.0(79)$ & $2.1(32)$ & $3.5(50)$ & $2.42(0.008)$ \\
\hline ICD-11 complex PTSD class & $1.0(26)$ & $0.6(9)$ & $1.3(18)$ & $1.97(0.024)$ \\
\hline
\end{tabular}

Estimates weighted for over-sampling of child protection cases

$Z$ test comparing proportions of males and females

Significant differences in bold

Table 2 Frequency of exposure to different forms of traumatic stressors for the total trauma-exposed sample, and males and females

\begin{tabular}{|c|c|c|c|c|}
\hline Traumatic stressors & $\begin{array}{l}\text { Total sample } \\
(N=2591) \\
\%(n)\end{array}$ & $\begin{array}{l}\text { Males }(N=1346) \\
\%(n)\end{array}$ & $\begin{array}{l}\text { Females }(N=1245) \\
\%(n)\end{array}$ & $Z(p)$ \\
\hline Death of close family member & $71(1839)$ & $66.2(891)$ & 76.1 (947) & $5.53(<0.001)$ \\
\hline Road traffic accident & $10.6(275)$ & $12.1(163)$ & $9.0(112)$ & $2.57(0.005)$ \\
\hline Near-drowning & $7.2(187)$ & $9.1(123)$ & $5.2(65)$ & $3.84(<0.001)$ \\
\hline Victim of robbery & $8.8(228)$ & $9.5(128)$ & $8.0(100)$ & $1.33(0.092)$ \\
\hline Adult physical assault ( $>18$ years) & $16.8(436)$ & $20.0(268)$ & $13.5(167)$ & $4.42(<0.001)$ \\
\hline Childhood physical assault ( $<18$ years) & $48.7(1263)$ & $52.5(706)$ & 44.7 (557) & $3.92(<0.001)$ \\
\hline Childhood bullying ( $<18$ years) & $44.4(1150)$ & $41.9(563)$ & $47.3(587)$ & $2.72(0.003)$ \\
\hline Childhood sexual abuse ( $<18$ years) & $2.5(66)$ & $0.7(9)$ & $4.6(57)$ & $6.31(<0.001)$ \\
\hline
\end{tabular}

Estimates weighted for over-sampling of child protection cases

$Z$ test comparing proportions of males and females

Significant differences in bold

and childhood bullying. Notably, a small percentage of the sample reported experiencing childhood sexual abuse (2.5\%). Statistically significant sex differences were observed for all trauma types except being the victim of a robbery. A significantly greater proportion of females experienced the death of a close family member, childhood bullying, and childhood sexual abuse. Contrastingly, a significantly greater proportion of males experienced a road traffic accident, near-drowning, physical assault during adulthood, and physical assault during childhood.

\section{Associations between traumatic exposure and PTSD and CPTSD class membership}

Inspection of the unadjusted ORs in Table 3 shows that five of the eight traumatic exposures were associated with an increased risk of PTSD class membership (as compared to the non-symptomatic class). The strongest associations were observed between childhood sexual abuse and adult physical assault. Females were also significantly more likely than males to belong to the PTSD class. With respect to CPTSD class membership 
Table 3 Chi square unadjusted odds ratios between traumatic events, covariates, and PTSD and CPTSD class membership ( $N=2591)$

\begin{tabular}{|c|c|c|c|c|c|c|}
\hline Variable & $\begin{array}{l}\text { ICD-11 PTSD } \\
\text { OR (95\% CI) }\end{array}$ & $P$ & $\begin{array}{l}\text { ICD-11 CPTSD } \\
\text { OR }(95 \% \mathrm{CI})\end{array}$ & $P$ & $\begin{array}{l}\text { ICD-11 CPTSD* } \\
\text { OR }(95 \% \text { CI })\end{array}$ & $P$ \\
\hline Death of a close family member & $1.30(0.81 / 2.10)$ & 0.311 & $1.04(0.43 / 2.51)$ & 1.00 & $1.36(0.51 / 3.66)$ & 0.630 \\
\hline Road traffic accident & $1.54(0.82 / 2.89)$ & 0.190 & $1.56(0.54 / 4.57)$ & 0.342 & $1.02(0.30 / 3.47)$ & 1.00 \\
\hline Near-drowning & $2.21(1.15 / 4.26)$ & $\mathbf{0 . 0 2 3}$ & $2.45(0.83 / 7.18)$ & 0.104 & $1.11(0.32 / 3.83)$ & 1.00 \\
\hline Robbery & $2.15(1.16 / 3.96)$ & 0.022 & $1.40(0.42 / 4.70)$ & 0.483 & $1.17(0.28 / 4.81)$ & 0.828 \\
\hline Adult physical assault & $3.37(2.11 / 5.38)$ & 0.000 & $10.17(4.50 / 22.99)$ & 0.000 & $3.02(1.20 / 7.64)$ & 0.023 \\
\hline Childhood physical assault & $1.40(0.89 / 2.20)$ & 0.168 & $5.93(2.04 / 17.25)$ & 0.000 & $4.25(1.34 / 13.50)$ & 0.010 \\
\hline Childhood bullying & $1.57(1.00 / 2.48)$ & $\mathbf{0 . 0 5 0}$ & $3.30(1.37 / 7.92)$ & 0.007 & $2.09(0.79 / 5.58)$ & 0.165 \\
\hline Childhood sexual abuse & $3.84(1.60 / 9.24)$ & 0.008 & $24.76(10.55 / 58.13)$ & 0.000 & $6.44(2.02 / 20.55)$ & 0.002 \\
\hline Sex (female) & $1.71(1.08 / 2.71)$ & 0.022 & $2.09(0.93 / 4.71)$ & 0.077 & $1.22(0.48 / 3.08)$ & 0.817 \\
\hline Living status & $1.04(0.63 / 1.72)$ & 1.00 & $1.34(0.54 / 3.36)$ & 0.665 & $1.29(0.46 / 3.63)$ & 0.799 \\
\hline Employment status & $1.89(0.89 / 4.00)$ & 0.133 & $10.31(4.60 / 23.14)$ & 0.000 & $5.47(1.86 / 16.05)$ & 0.002 \\
\hline
\end{tabular}

Significant effects in bold

Estimates weighted for over-sampling of child protection cases

Reference group non-symptomatic class, OR (95\% CI) Odds Ratio with 95\% confidence interval, $P$ statistical significance value

*In this set of analysis, the PTSD class was set as the reference category

Table 4 Multinomial logistic regression adjusted odds ratios between traumatic events, covariates, and PTSD and CPTSD class membership $(N=2591)$

\begin{tabular}{|c|c|c|c|c|c|c|}
\hline Variable & $\begin{array}{l}\text { ICD-11 PTSD } \\
\text { OR }(95 \% \text { CI })\end{array}$ & $P$ & $\begin{array}{l}\text { ICD-11 CPTSD } \\
\text { OR }(95 \% \mathrm{CI})\end{array}$ & $P$ & $\begin{array}{l}\text { ICD-11 CPTSD* } \\
\text { OR }(95 \% \mathrm{CI})\end{array}$ & $P$ \\
\hline Death of a close family member & $1.32(0.82 / 2.15)$ & 0.258 & $1.01(0.40 / 2.57)$ & 0.983 & $1.31(0.47 / 3.68)$ & 0.608 \\
\hline Road traffic accident & $1.32(0.82 / 2.15)$ & 0.418 & $1.25(0.37 / 4.20)$ & 0.719 & $1.25(0.47 / 4.70)$ & 0.944 \\
\hline Near-drowning & $2.08(1.06 / 4.12)$ & 0.034 & $1.62(0.47 / 5.56)$ & 0.441 & $1.05(0.27 / 4.02)$ & 0.718 \\
\hline Robbery & $1.85(0.98 / 3.48)$ & 0.059 & $1.58(0.43 / 5.81)$ & 0.491 & $1.17(0.28 / 4.81)$ & 0.828 \\
\hline Adult physical assault & $3.19(1.96 / 5.18)$ & 0.000 & $6.80(2.84 / 16.13)$ & 0.000 & $2.13(0.80 / 5.65)$ & 0.128 \\
\hline Childhood physical assault & $1.23(0.78 / 1.97)$ & 0.372 & $3.62(1.21 / 10.87)$ & 0.021 & $2.92(0.90 / 9.52)$ & 0.074 \\
\hline Childhood bullying & $1.39(0.87 / 2.21)$ & 0.167 & $2.04(0.81 / 5.13)$ & 0.132 & $1.47(0.53 / 4.05)$ & 0.462 \\
\hline Childhood sexual abuse & $1.90(0.73 / 4.95)$ & 0.186 & $9.43(3.41 / 26.32)$ & 0.000 & $4.98(1.35 / 18.52)$ & 0.016 \\
\hline Sex (female) & $2.00(1.23 / 3.24)$ & 0.005 & $2.02(0.80 / 5.08)$ & 0.138 & $1.01(0.36 / 2.74)$ & 0.987 \\
\hline Living status & $1.04(0.63 / 1.75)$ & 0.855 & $1.23(0.44 / 3.40)$ & 0.691 & $1.17(0.38 / 3.58)$ & 0.781 \\
\hline Employment status & $1.66(0.77 / 3.61)$ & 0.197 & $6.99(2.77 / 17.54)$ & 0.000 & $4.20(1.33 / 13.33)$ & 0.015 \\
\hline
\end{tabular}

Significant effects in bold

Estimates weighted for over-sampling of child protection cases

Reference group non-symptomatic class, OR (95\% CI) Odds Ratio with 95\% confidence interval, $P$ statistical significance value

*In this set of analysis, the PTSD class was set as the reference category

(as compared to the non-symptomatic class), the unadjusted ORs indicated a significant relationship between four traumatic events and CPTSD. All three childhood exposures were significantly associated, along with adult physical assault, and a strong association was observed for childhood sexual abuse. Unemployment status was also significantly, and robustly, associated with CPTSD class membership. Finally, those who had experienced childhood sexual abuse, childhood physical assault, adult physical assault, and were unemployed were significantly more likely to be classified with CPTSD as compared to PTSD.

Table 4 reports the adjusted ORs from the multinomial logistic regression analyses. The model which contained sex, living status, employment status, and the eight traumatic stressors was statistically significant $\left(\chi^{2}\right.$ $(22)=132.67, p<.001)$. Within this multivariate model, two traumatic events remained significant predictors of 
PTSD class membership (as compared to the non-symptomatic class): adult physical assault, and experiencing a near-drowning. Sex remained significant with females twice as likely as males to belong to the PTSD class. With respect to CPTSD class membership (as compared to the non-symptomatic class), childhood bullying was no longer a significant predictor. Childhood sexual abuse was the strongest predictor of CPTSD class membership, followed by unemployment status, adult physical assault, and childhood physical assault. In terms of differentiating CPTSD class membership from PTSD class membership, two variables emerged as significant predictors. Those who had experienced childhood sexual abuse were approximately five times more likely to belong to the CPTSD class than the PTSD class, and those who were unemployed were approximately four times more likely to belong to the CPTSD class than the PTSD class.

Finally, to determine the effect of cumulative exposure to multiple forms of childhood interpersonal trauma on risk of CPTSD classification, as compared to PTSD classification an aggregate childhood trauma variable was constructed $(0,1,2$, and 3$)$. This aggregate variable was entered into the equation as a categorical variable and the first level (zero childhood interpersonal traumatic exposures) was used as the reference category for a simple contrast. The model as a whole was statistically significant $\left(\chi^{2}(11)=29.95, p=.002\right)$. The odds ratios for one childhood interpersonal trauma $(\mathrm{OR}=2.00, p=.51,95 \%$ $\mathrm{CI}=0.26-15.53)$, two traumas $(\mathrm{OR}=2.57, p=.37,95 \%$ $\mathrm{CI}=0.33-19.82)$, and three traumas $(\mathrm{OR}=77.66, p=.005$, 95\% $\mathrm{CI}=3.67-1645.78)$ indicated an increasing risk of CPTSD classification, as compared to PTSD classification, as the number of childhood traumatic exposures increased.

\section{Discussion}

The ICD-11 proposals for PTSD and CPTSD suggest that exposure to traumatic stressors of an interpersonal nature which are prolonged and repeated, or comprised of multiple forms under conditions from which escape is difficult or impossible, are likely to increase risk of CPTSD as opposed to PTSD. In particular, interpersonal trauma experiences that occur during early development are regarded as prototypical forms of trauma that are likely to increase risk of DSO symptomatology, in addition to core PTSD symptomatology. The objective of the current study was to determine if exposure to certain forms of interpersonal trauma during the first 18 years of life (sexual abuse, physical assault, and bullying) was associated with an elevated risk of CPTSD symptomatology, as compared to PTSD, and if exposure to multiple forms of childhood interpersonal trauma was associated with an elevated risk of CPTSD symptomatology, as compared to PTSD symptomatology, in a dose-response fashion.

The majority $(87.1 \%)$ of Danes aged 24 were found to have been exposed to at least one of the eight traumatic life events measured in this study, thus satisfying the traumaticexposure requirement in ICD-11 for consideration of a diagnosis of PTSD or CPTSD. Findings from the WHO's World Mental Health Survey Consortium [26] which analysed epidemiological data (primarily of a nationally representative nature) from 24 nations found that, collectively, $70.4 \%$ of individuals had experienced at least one lifetime traumatic exposure. The range of traumatic exposure varied considerably from a low of $28.6 \%$ in Bulgaria to a high of $84.6 \%$ in Ukraine. Denmark was not included within these national figures; therefore, current findings suggest that this cohort of Danes aged 24 is reflective of the higher end of the global continuum of traumatic exposure.

The trauma literature has consistently indicated that PTSD is a more common disorder among females, with a two-fold increased prevalence as compared to males [27, 28]. In the current study, bivariate results were consistent with the existing literature indicating that females were twice as likely as males to belong to the PTSD and CPTSD classes. Within the multivariate analyses sex remained a significant predictor of PTSD class membership but did not significantly predict CPTSD classification. It was notable, however, that the effect sizes for the relationship between sex and both PTSD and CPTSD class membership were equivalent suggesting that the null effect for sex on CPTSD classification was likely the result of insufficient statistical power due to the small number of people who belonged to the CPTSD class. Although sex appeared to be associated with an increased risk of both PTSD and CPTSD classification when compared to the non-symptomatic class, sex did not serve to distinguish CPTSD classification from PTSD classification specifically. In other words, being female appears to be associated with an increased risk of PTSD and CPTSD, even when controlling for various forms of traumatic exposure that predominately effect females (e.g., childhood sexual abuse), however sex does appear to differentiate between a PTSD and CPTSD response to trauma. To extend work regarding the role of sex in the prediction of both PTSD and CPTSD, it will be important that future work considers the interaction effects between sex and traumatic exposure.

Results of the bivariate and multivariate analyses were generally supportive of proposals for ICD-11 and in-line with prior findings [7]. Within the multivariate framework, controlling for different forms of trauma and sociodemographic risk-factors, childhood sexual abuse was the strongest risk factor for CPTSD classification, as compared to the non-symptomatic class. Furthermore, exposure to physical assault during both childhood and adulthood increased 
risk of CPTSD classification. In an attempt to differentiate CPTSD class membership from PTSD class membership, the only traumatic experience that remained significant within the multivariate model was childhood sexual abuse. Furthermore, results indicated that cumulative exposure to childhood interpersonal trauma heightened risk of CPTSD symptomatic responses, as compared to PTSD symptomatic responses, in a manner possibly suggestive of a dose-response relationship; the small sample sizes and wide confidence intervals should, however, suggest caution against over-interpreting these result. Nonetheless, these results contribute to existing findings [7, 22] supporting the ICD-11 proposals that exposure to interpersonal abuse during early development leads to an increased likelihood of a complex psychological response to trauma.

That childhood sexual abuse, and the accumulation of early interpersonal forms of trauma, conferred an increased risk for CPTSD symptomatic responses over and above PTSD symptoms, suggests that the DSO symptom categories that characterise CPTSD may be specifically interpretable in the context of these particular forms of trauma. For instance, evidence from the trauma and developmental psychopathology literature suggests that early interpersonal trauma/abuse, particularly of a sexual nature, can directly promote and facilitate negative self-concept formulation. Multiple studies attest to this trauma induced 'self-criticism'. For example, childhood trauma (especially interpersonal and sexual abuse) has been shown to be highly associated with 'mental contamination' [29], self-denigration [30], self-disgust [31], self-harm [32] and suicidality [33]. Given the context and nature of these specific early traumatic stressors, CPTSD may capture those specific selfevaluative features that reflect the trauma-induced shame, guilt, depression, and disgust commonly associated with these early forms of interpersonal trauma.

Regarding the disturbed relationship cluster of CPTSD, evidence shows that childhood interpersonal traumas such as sexual abuse often create an enduring vulnerability which is accompanied, facilitated, and compounded by social withdrawal, disconnection, and isolation [34]. Commonly identified in sexually traumatised individuals [35, 36], social inhibitors, and constraints such as avoidance, social anxiety, and social phobia may plausibly exacerbate and compound posttraumatic reactions and in turn facilitate and promote a more 'complex' manifestation of PTSD. The distinctiveness of the CPTSD construct and the greater impairment associated with it (as compared to PTSD: see Elklit et al. [23]) may therefore be partly attributable to the often extreme and negative social and socializing consequences of these early forms of interpersonal threat, violation, and harm.

Finally, regarding affect dysregulation symptoms, the trauma literature is replete with studies that evidence the impaired ability of childhood sexual trauma survivors to regulate and/or tolerate negative emotional states (see Dvir et al. [10]). Childhood interpersonal trauma, especially repeated and prolonged traumatic exposures that are expected to increase risk of CPTSD, often compromises the acquisition and development of 'appropriate' affective regulatory skills and can thus be meaningfully understood in relation to the affective dysregulation symptoms within a CPTSD framework. This disruption of emotional regulation may occur as a direct result of the psychological impact of the trauma but may also potentially reflect the well-evidenced neurobiological effects of such early interpersonal trauma [37-39]. Collectively, therefore, these three DSO categories that are proposed to distinguish CPTSD from PTSD certainly 'fit' the existing evidence base that currently informs our understanding of the physical, affective, behavioural, cognitive, and interpersonal sequelae that characterise these early forms of interpersonal trauma.

A number of important limitations should be considered when interpreting the current findings. First, a weakness of the study was the approximation of the PTSD and DSO symptoms. The PTSD symptoms were not anchored to an index trauma, and the DSO symptoms were predominately derived from non-clinical scales. The problematic nature of use of items from non-clinical scales is evidenced by the very high rates of endorsement for the three DSO symptom clusters. This suggests that although estimates of ICD11 PTSD and CPTSD were low (3 and 1\%, respectively), these figures are likely inflated relative to the true cases of PTSD and CPTSD. Nationally representative surveys using measures specifically designed to capture ICD-11 PTSD and CPTSD are thus warranted. Second, the most commonly endorsed traumatic life event within this cohort was experiencing the death of a loved one; an event that is more closely related to the experience of prolonged grief disorder (PGD) than PTSD and CPTSD [40]. The inability to screen for prolonged grief reactions likely limited our capacity to fully understand the mental health effects of the traumatic events encountered within this sample. Future work will need to explore the interplay between PTSD, CPTSD, and PGD, and will need to consider whether exposure to the death of a loved one can be considered a traumatic event that allows one to qualify for a diagnosis of PTSD or CPTSD, as opposed to PGD.

Despite these limitations, the current study provides novel and important evidence to inform the psychotraumatology research literature. The findings indicate that exposure to early developmental trauma is meaningfully associated with CPTSD symptomatology, and that exposure to childhood sexual abuse, specifically, predicts a complex traumatic response as compared to a PTSD response. Additionally, in-line with ICD-11 proposals for CPTSD, we also found tentative evidence that exposure to multiple forms of 
early developmental trauma increases risk of CPTSD in a dose-response manner. It was also notable that unemployment status was also a meaningful differentiating factor between CPTSD and PTSD suggesting that social factors play an important role in understanding the development of CPTSD. Trauma history appears to be important in understanding the development of CPTSD but researchers should not ignore the wider societal context in which the trauma, and the traumatic response, occurs. Current findings add to a growing literature supporting the validity of CPTSD as a unique diagnostic entity. This has important clinical implications as CPTSD, possessing a distinct aetiology and symptom composition, likely requires distinct clinical interventions to maximise treatment efficacy [41].

Author contribution statement PH, JM, MS, FV, EM, and MC (Cloitre) were responsible for the development of the study objectives, study design, and analytical strategy. All contributed to conducting the statistical analysis, background research, and writing of the manuscript. AE and MC (Christoffersen) were responsible for gaining access to the data used in this study, and were responsible for writing the method section of manuscript. Both contributed to the manuscript as a whole through revisions. All authors contributed meaningfully to the production of the manuscript submitted for review, and have agreed to the order of authors as presented.

\section{Compliance with ethical standards}

Conflict of interest Marylène Cloitre participated as a member of the World Health Organization Working Group on the Classification of Disorders Specifically Associated with Stress, reporting to the International Advisory Group for the Revision of ICD-10 Mental and Behavioural Disorders. However, the views expressed reflect the opinions of the author and not necessarily the Working Group or Advisory Group and the content of this article does not represent WHO policy. No conflict of interest associated with other authors.

\section{References}

1. Maercker A, Brewin CR, Bryant RA, Cloitre M, Reed GM, van Ommeren M, Saxena S (2013) Proposals for mental disorders specifically associated with stress in the ICD-11. Lancet 381:1683-1685. doi:10.1016/S0140-6736

2. First MB, Reed GM, Hyman SE, Saxena S (2015) The development of the ICD-11 clinical descriptions and diagnostic guideline for mental and behavioural disorders. World. Psychiatry 14:82-90. doi:10.1002/wps.20189

3. Brewin CB, Lanius RA, Nova A, Schnyder U, Galea S (2009) Reformulating PTSD for DSM-V: life after criterion A. J Trauma Stress 22:366-373. doi:10.1002/jts.20443

4. Herman JL (1992) Complex PTSD: a syndrome in survivors of prolonged and repeated trauma. J Trauma Stress 5:377-391
5. van der Kolk BA, Roth S, Pelcovitz D, Sunday S, Spinazzola J (2005) Disorders of extreme stress: the empirical foundation of a complex adaptation to trauma. J Trauma Stress 18:389-399. doi:10.1002/jts. 20047

6. Cloitre M, Courtois CA, Charuvastra A, Carapezza R, Stolbach BC, Green BL (2011) Treatment of complex PTSD: results of the ISTSS expert clinician survey on best practices. J Trauma Stress 24:615-627. doi:10.1002/jts.20697

7. Cloitre M, Garvert DW, Brewin CR, Bryant RA, Maercker A (2013). Evidence for proposed ICD-11 PTSD and complex PTSD: a latent profile analysis. Eur J Psychotraumatol 4:20706. doi:10.3402/ejpt.v4i0.20706

8. Hyland P, Shevlin M, Elklit A, Murphy J, Vallières F, Garvert DW, Cloitre M (2016) An assessment of the construct validity of the ICD-11 proposals for complex posttraumatic stress disorder. Psychol Trauma. doi:10.1037/tra0000114

9. Cloitre M, Stolbach BC, Herman JL, van der Kolk B, Pynoos R, Wang J, Petkova E (2009) A developmental approach to complex PTSD: childhood and adult cumulative trauma as predictors of symptom complexity. J Trauma Stress 22:399-408. 10.1002/ jts. 20444

10. Dvir Y, Ford JD, Hill M, Frazier JA (2014) Childhood maltreatment, emotional dysregulation, and psychiatric comorbidities. Harv Rev Psychiatry 22:149-161. doi:10.1097/ HRP.0000000000000014

11. Pratchett LC, Yehuda R (2011) Foundations of posttraumatic stress disorder: does early life trauma lead to adult posttraumatic stress disorder? Dev Psychopathol 23:477-491. doi:10.1017/ S0954579411000186

12. Shipman K, Edwards A, Brown A, Swisher L, Jennings E (2005) Managing emotion in a maltreating context: a pilot study examining child neglect. Child Abuse Negl 29:1015-1029

13. Cloitre M, Scarvalone P, Difede J (1997) Post-traumatic stress disorder, self and interpersonal dysfunction among sexually retraumatized women. J Trauma Stress 10:435-450. doi:10.102 3/A:1024893305226

14. Finkelhor D, Turner H, Ormrod R, Hamby SL (2009) Violence, abuse, and crime exposure in a national sample of children and youth. Pediatrics 124:1411-1423

15. Finkelhor D, Turner H, Shattuck A, Hamby SL (2013). Violence, abuse, and crime exposure in a national sample of children and youth: an update. JAMA Pediatr 167:614-621. doi:10.1001/ jamapediatrics.2013.42.

16. Cloitre M, Garvert DW, Weiss B, Carlson EB, Bryant RA (2014). Distinguishing PTSD, complex PTSD, and borderline personality disorder: a latent class analysis. Eur J Psychotraumatol 5:25097. doi:10.3402/ejpt.v5.25097

17. Wolf EJ, Miller MW, Kilpatrick D, Resnick HS, Badour CL, Marx BP, Keane TM, Rosen RC, Friedman MJ (2014) ICD11 complex PTSD in US national and veteran samples: prevalence and structural associations. Clinic Psychol Sci 2:215-229. doi: $10.1177 / 2167702614545480$

18. Perkonigg A, Hofler M, Cloitre M, Wittchen HU, Trautmann S, Maercker A (2016) Evidence for two different ICD-11 posttraumatic stress disorder in a community sample of adolescents and young adults. Eur Arch Psychiatry Clin Neurosci 266:317-328. doi:10.1007/s00406-015-0639-4

19. Forbes D, Lockwood E, Creamer M, Bryant RA, McFarlane AC, Silove D, Nickerson A, O'Donnell M (2015). Latent structure of the proposed ICD-11 post-traumatic stress disorder symptoms: implications for the diagnostic algorithm. Br J Psychiatr 206:245-251. doi:10.1192/bjp.bp.114.150078

20. Hansen M, Hyland P, Armour C, Elklit A, Shevlin, M (2015). Less is more? Assessing the validity of the ICD-11 model of PTSD across multiple trauma samples. Eur J Psychotraumatol 6:28766. doi:10.3402/ejpt.v6.28766 
21. Tay, AK, Rees S, Chen J, Kareth M, Silove D (2015). The structure of post-traumatic stress disorder and complex post-traumatic stress disorder amongst West Papuan refugees. BMC Psychiat 15:111. doi:10.1186/s12888-015-0480-3

22. Knefel M, Lueger-Schuster B (2013). An evaluation of ICD-11 PTSD and complex PTSD criteria in a sample of adult survivors of childhood institutional abuse. Eur J Psychotraumatol 4:22608, doi:10.3402/ejpt.v4i0.22608

23. Elklit A, Hyland P, Shevlin M (2014). Evidence of symptom profiles consistent with posttraumatic stress disorder and complex posttraumatic stress disorder in different trauma samples. Eur J Psychotraumatol 5:24221. doi:10.3402/ejpt.v5.24221

24. Knefel M, Garvert DW, Cloitre M, Lueger-Schuster B (2015). Update to an evaluation of ICD-11 PTSD and complex PTSD criteria in a sample of adult survivors of childhood institutional abuse by Knefel \& Lueger-Schuster (2013): a latent profile analysis. Eur J Psychotraumatol 6:25290. doi:10.3402/ejpt.v6.25290

25. Rosenberg M (1965) Society and the adolescent self-image. Princeton University Press, Princeton

26. Benjet C, Bromet E, Karam EG, Kessler RC, McLaughlin KA, Ruscio AM, Shahly V, Stein DJ, Petukhova M, Hill E, Koenen RC (2016) The epidemiology of traumatic event exposure worldwide: results from the World Mental Health Survey Consortium. Psychol Med 46:327-343. doi:10.1017/S0033291715001981

27. Christiansen DM, Hansen M (2015) Accounting for sex differences in PTSD: A multi-variable mediation model. Eur J Psychotraumatol 6:26068. doi:10.3402/ejpt.v6.26068

28. Kessler RC, Sonnega A, Bromet E, Hughes M, Nelson CB (1995). Posttraumatic stress disorder in the National Comorbidity Survey. Arc Gen Psychiat 52:1048-1060.

29. Badour CL, Adams TG (2015) Contaminated by trauma: understanding links between self-disgust, mental contamination, and PTSD. In: Overton P, Simpson J, Powell P. (eds) The revolting self: Psychological and clinical perspectives on self-directed disgust. Karnac Books, London, pp 127-149

30. Gilbert P (2015) Self-disgust, self-hatred, and compassionfocused therapy. In: Overton P, Simpson J, Powell P. (eds) The revolting self: Psychological and clinical perspectives on selfdirected disgust. Karnac Books, London, pp 223-243

31. Overton PG, Powell PA, Simpson J (2015) The revolting self: Perspectives on the psychological, social, and clinical implications of self-directed disgust. Karnac Books, London
32. Saçarçelik G, Türkcan A, Güveli H, Yeşilbaş D, Can İÖ, Uyanıker ZD, Altınbaş, K (2013). Evaluation of the relation between deliberate self-harm behavior and childhood trauma experiences in patients admitted to the second-step psychiatric outpatient clinic for adolescent and young adult. Arch Neuropsychiatry 50:222-229. doi:10.4274/npa.y6257

33. Park S, Hong JP, Jeon HJ, Seong S, Cho MJ (2015). Childhood exposure to psychological trauma and the risk of suicide attempts: the modulating effect of psychiatric disorders. Psychiatry Investig 12:171-176.

34. Johnson DM, Sheahan TC, Chard KM (2003) Personality disorders, coping strategies, and posttraumatic stress disorder in women with histories of childhood sexual abuse. J Child Sex Abuse 12:19-39

35. Bal S, Van Oost P, De Bourdeaudhuij I, Crombez G (2003) Avoidant coping as a mediator between self-reported sexual abuse and stress-related symptoms in adolescents. Child Abuse Negl 27:883-897

36. Walsh K, Fortier MA, DiLillo D (2010) Adult coping with childhood sexual abuse: a theoretical and empirical review. Aggress Violent Behav 15:1-13.

37. Burns EE, Jackson JL, Harding HG (2010) Child maltreatment, emotion regulation, and posttraumatic stress: the impact of emotional abuse. J Aggress Maltreat Trauma 19:801-819

38. Cicchetti D, Rogosch FA (2009) Adaptive coping under conditions of extreme stress: multilevel influences on the determinants of resilience in maltreated children. New Dir Child Adolesc Dev 124:47-59

39. De Bellis MD (2001) Developmental traumatology: the psychobiological development of maltreated children and its implications for research, treatment, and policy. Dev Psychopathol 13:539-564

40. Maciejewski PK, Maercker A, Boelen PA, Prigerson HG (2016) "Prolonged grief disorder" and "persistent complex bereavement disorder", but not "complicated grief", are one and the same diagnostic entity: an analysis of data from the Yale Bereavement Study. World Psychiatry 15:266-275. doi:10.1002/wps.20348

41. Ford JD (2015) Complex PTSD: research directions for nosology/assessment, treatment, and public health. Eur J Psychotraumatol 6, 27584. 10.3402/ejpt.v6.27584 